\title{
Review
}

\section{Endothelial Cell Metabolism in Health and Disease}

\author{
Katerina Rohlenova, ${ }^{1,2,4}$ Koen Veys, ${ }^{1,2,4}$ Ines Miranda-Santos, ${ }^{1,2}$ Katrien De Bock, ${ }^{3}$ and \\ Peter Carmeliet ${ }^{1,2, *}$
}

The metabolism of endothelial cells (ECs) has only recently been recognized as a driving force of angiogenesis. Metabolic pathways, such as glycolysis, fatty acid oxidation, and glutamine metabolism, have distinct, essential roles during vessel formation. Moreover, EC metabolism is markedly perturbed in pathologies such as cancer and diabetes. For instance, because tumor ECs increase glycolysis, lowering hyperglycolysis in tumor ECs induces therapeutic benefits in preclinical tumor models. Expanding our knowledge of how ECs alter their metabolism in disease could pave the way for novel therapeutic opportunities. In this review, we discuss the most recent insights into EC metabolism in health and disease, with emphasis on the changes in metabolism in the tumor endothelium.

\section{Angiogenesis: From Quiescent Cells to a New Vessel Sprout}

ECs form the inner lining of blood and lymphatic vessels and are essential for normal functioning of the vascular system. The vascular network expands in response to changing metabolic demands during physiological organ growth to supply all tissues with sufficient oxygen and nutrients. Throughout adulthood, ECs stay mostly quiescent; however, they retain the capacity to rapidly initiate new vessel formation in response to injury or in pathological conditions. This tightly regulated process, called 'sprouting angiogenesis', starts with the protrusion of highly motile filopodia from a migratory tip cell and continues as proliferating stalk cells trail behind the leading tip cell as the sprout elongates [1] (Figure 1A). The phenotype of individual EC subtypes is not hard-wired. Instead, it is dynamically adjusted in response to cellular competition for growth factors, with vascular endothelial growth factor (VEGF) being a key regulator [2]. As the newly formed vessel matures, the restored perfusion contributes to the induction of a quiescent phalanx cell monolayer of tightly organized cobblestone-like cells. The three EC subtypes (tip, stalk, and phalanx cells) differ in their energy, biomass, and redox requirements, and, thus, in their metabolism $[3,4]$. A growing body of evidence indicates that these metabolic changes are not mere bystanders during the angiogenic switch. Instead, it is increasingly evident that growth factors and transcriptional signals converge on metabolic changes that drive phenotypic differentiation [3]. Recent evidence suggests that EC metabolism is perturbed in diseased tissues, such as in tumors and during diabetes. Therefore, deeper insight into metabolic alterations in ECs in these pathological conditions is required to exploit the translational potential of normalizing these metabolic perturbations.

\section{EC Metabolism in Health}

Glycolysis Is the Main Energy Source in the Endothelium

ECs are glycolysis addicts, since $85 \%$ of their ATP is produced glycolytically by converting glucose to lactate [3]. Compared with many other healthy cell types, ECs have higher rates of
Trends

EC metabolism determines vessel sprouting; thus, targeting EC metabolism offers new therapeutic opportunities to inhibit or stimulate vessel formation.

Tumor vessel normalization by targeting endothelial metabolism improves combination chemotherapy and reduces metastasis.

Cooling the overheated metabolic engine of tumor ECs to normal levels may be an effective, yet safe strategy, and may be superior to completely eliminating the perturbed metabolic pathways.

${ }^{1}$ Laboratory of Angiogenesis and Vascular Metabolism, VIB Center for Cancer Biology, VIB, Leuven, Belgium ${ }^{2}$ Laboratory of Angiogenesis and Vascular Metabolism, Department of Oncology, KU Leuven, Leuven, Belgium

${ }^{3}$ Laboratory of Exercise and Health, Department of Health Sciences and Technology, ETH Zurich, Zurich, Switzerland

${ }^{4}$ These authors contributed equally

*Correspondence:

peter.carmeliet@kuleuven.vib.be (P. Carmeliet). 


\section{Trends in Cell Biology}
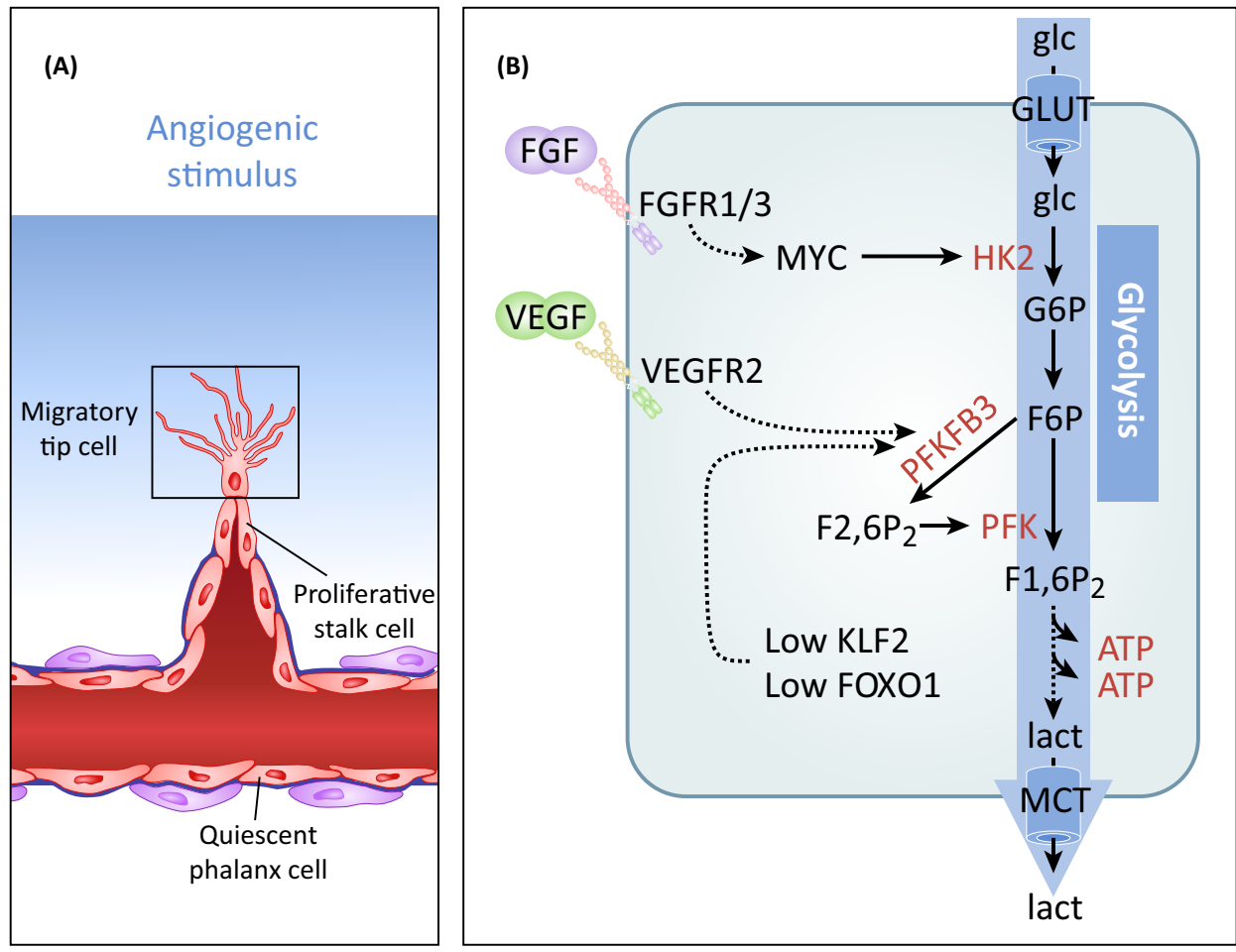

Trends in Cell Biology

Figure 1. Glycolysis: The Main Energy Source in Sprouting Endothelium. (A) The migratory tip cell initiates the formation of a sprout from an existing quiescent vessel upon an angiogenic stimulus. Proliferative stalk cells follow the tip cell and eventually create a new vessel sprout. (B) VEGF and FGF promote glycolysis by induction of PFKFB3 and by MYCmediated stimulation of HK2. High levels of glycolysis provide ATP to support the migration of the tip cell towards the angiogenic stimuli. At the same time, the levels of FOXO1 and KLF2, markers of quiescent endothelial cells (ECs), are repressed. Abbreviations: $F 1,6 P_{2}$, fructose-1,6-bisphosphate; $F 2,6 P_{2}$, fructose-2,6-bisphosphate; F6P, fructose-6phosphate; FGF, fibroblast growth factor; FGFR1/3, fibroblast growth factor receptor 1/3; FOXO1, forkhead box O1; G6P, glucose-6-phosphate; glc, glucose; GLUT, glucose transporter; HK2, hexokinase 2; KLF2, Krüppel-like factor; lact, lactate; MCT, monocarboxylate transporter; MYC, c-MYC; PFKFB3, phosphofructokinase-2/fructose-2,6-bisphosphatase 3; VEGF, vascular endothelial growth factor; VEGFR2, vascular endothelial growth factor receptor 2.

glycolysis and their glucose consumption is in the same range as that of many cancer cells [3]. Even though oxidative metabolism yields 34 extra molecules of ATP from one molecule of glucose, only a small fraction of pyruvate generated from glycolysis is metabolized oxidatively [3]. At first sight, it might seem enigmatic why quiescent ECs do not take full advantage of their easy access to oxygen. Phalanx cells bath in oxygen and, therefore, they might need to find ways to protect themselves from oxidative stress [5]. Preferentially utilizing glycolysis instead of oxidative metabolism would be one way of keeping reactive oxygen species (ROS) levels in check. At the same time, by relying on anaerobic metabolism, ECs save oxygen, which enhances the diffusion of oxygen to perivascular cells $[5,6]$. In addition, filopodia of tip cells explore and extend into hypoxic tissues, away from perfused blood vessels, where oxygen levels drop faster than glucose levels, making oxidative metabolism impractical. Also, glycolysis produces ATP with faster kinetics, necessary for the rapid revascularization of hypoxic tissues before their demise [3].

Glycolysis in ECs is stimulated by the regulator 6-phosphofructo-2-kinase/fructose-2,6bisphosphatase 3 (PFKFB3). This enzyme produces fructose-2,6-bisphosphate (F2,6P 2 , a 


\section{Trends in Cell Biology}

strong allosteric activator of phosphofructokinase-1 (PFK1), a rate-limiting enzyme of glycolysis [3]. Hexokinase 2 (HK2), the rate-limiting enzyme that phosphorylates glucose to glucose-6phosphate, is another glycolytic regulator in ECs [7]. Blood flow keeps phalanx cells in a resting state, partly through inhibition of glycolytic metabolism. PFKFB3, HK2, and other glycolytic genes are suppressed by activation of the transcription factor KLF2 upon laminar shear stress [8]. Forkhead box $\mathrm{O} 1$ (FOXO1) is another transcription factor that indirectly reduces glycolysis by inhibiting the transcription factor MYC $[9,10]$. When ECs switch from to angiogenic tip or stalk cells, their glycolytic rate is increased [3]. Angiogenic ECs can interchange their position in the sprout, depending on their relative metabolic fitness. Indeed, genetically instructed stalk cells can overtake the tip cell position when they express higher levels of PFKFB3 (and, thus, increase glycolysis) [3]. In addition to the genetic control of vascular sprouting, growth factordependent EC metabolism also controls angiogenesis. For instance, VEGF stimulates PFKFB3 levels, but reduced FOXO1 or KLF2 levels might also contribute to higher PFKFB3 levels during sprouting (Figure 1B). Of note, besides VEGF signaling, FGF signaling promotes MYC expression, thereby stimulating HK2 levels and glycolysis [7]. Hence, several other growth factor signaling pathways control metabolic pathways in ECs, such as glycolysis.

Interestingly, PFKFB3 and other glycolytic enzymes are compartmentalized in lamellipodia and filopodia to generate glycolytic ATP at so-called 'ATP hot-spots', colocalizing with F-actin in membrane ruffles of lamellipodia [3]. Reducing glycolysis by either pharmacological inhibition (with the small molecule 3PO) or genetic silencing of PFKFB3 decreased glycolytic rates in vitro, impairing EC proliferation and migration. Moreover, in vivo silencing of PFKFB3 (both pharmacologically and genetically) impaired vessel outgrowth and branching in the mouse retina and zebrafish [3]. Similarly, reducing glycolysis by silencing of HK2 reduced arterial development and branching in the mouse skin, as well as proliferation and number of filopodia in the retinal vasculature [7]. Altogether, these data underline the importance of glycolysis in angiogenesis and in the maintenance of EC specialization.

\section{Fatty Acids Support Biomass Synthesis}

In many cell types, mitochondria are the main site of ATP production, functioning as the energy powerhouse of the cell [11]. Acetyl-CoA molecules derived from fatty acids can be used in the TCA cycle to produce reducing equivalents to sustain ATP production via oxidative phosphorylation (OXPHOS). For example, cardiomyocytes primarily burn fatty acids to supply ATP for contractile purposes [12,13]. However, in ECs, mitochondria serve as a biosynthetic hub, rather than as an energy powerhouse [4]. Fatty acid oxidation (FAO)-derived carbons help to sustain the TCA cycle in conjunction with an anaplerotic substrate, resulting in the production of the precursors aspartate and glutamate from its intermediates oxaloacetate (OAA) and $\alpha$-ketoglutarate $(\alpha-K G)$ for deoxynucleotide (dNTP) synthesis during the proliferation of stalk cells [4] (Figure 2A). This is remarkable, because, in most other cells, acetyl-CoA is derived from glucose and/or glutamine to fuel the TCA cycle for biomass synthesis [11]. Carnitine palmitoyltransferase $1 \mathrm{a}(\mathrm{CPT} 1 \mathrm{a})$ is a rate-controlling enzyme of FAO that imports fatty acids into the mitochondria, where they are metabolized through $\beta$-oxidation into acetyl-CoA molecules that enter the TCA cycle [4]. CPT1a silencing causes a decrease in the dNTP pool, which perturbs EC sprouting in vitro (Figure 2B). Even though FAO is linked to redox homeostasis and ATP production in other cell types, the proliferation defect is not caused by redox imbalance or ATP depletion in ECs. However, a recent study documented that intracellular lipid droplets, formed upon loading with oleic acid, release fatty acids for energy production through FAO in ECs [14]. In vivo, genetic loss or pharmacological blockade of endothelial CPT1a reduced the number of branch points and diminished the radial expansion of the retinal vascular network in mice [4]. In 


\section{Trends in Cell Biology}

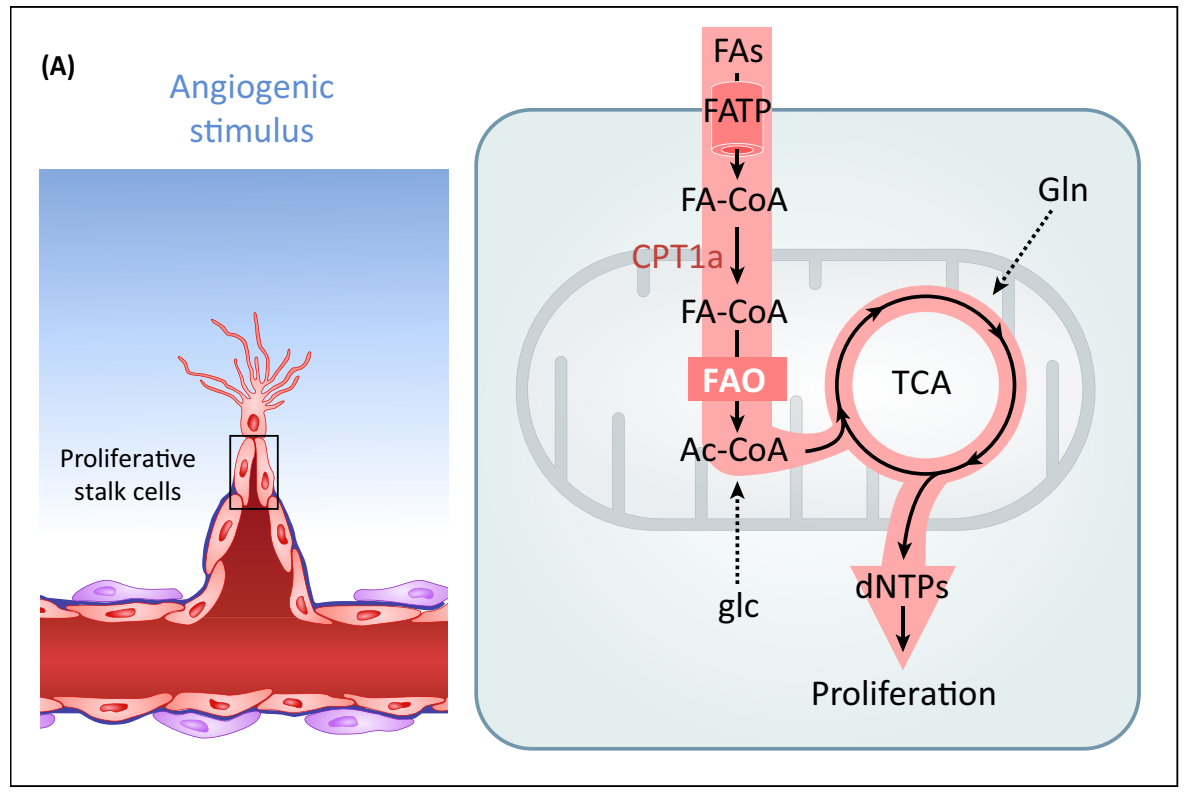

(B)

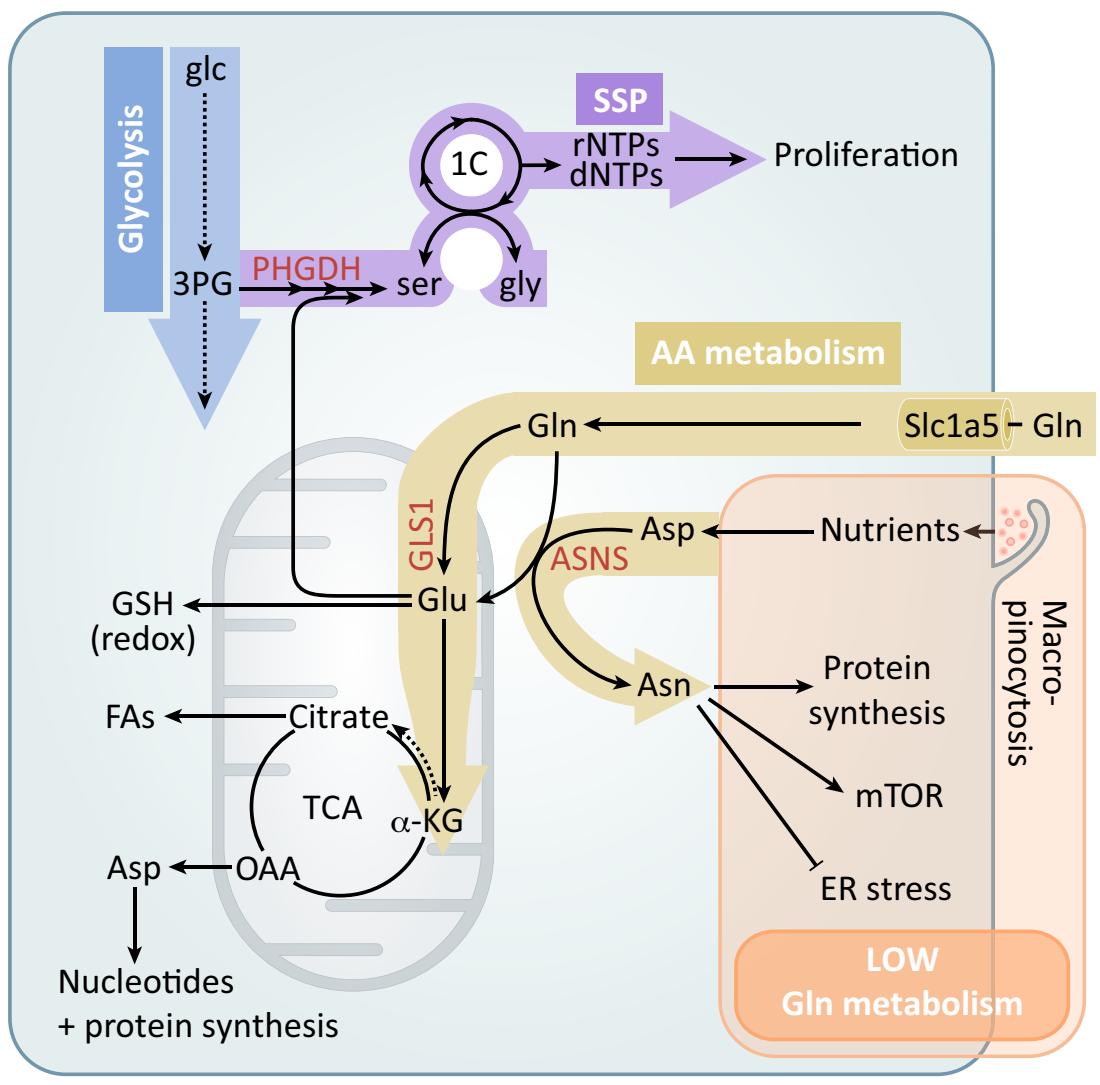

Trends in Cell Biology 


\section{Trends in Cell Biology}

accordance with the in vitro data, migratory properties were not affected, as evidenced by the normal number of filopodia [4]. Unlike PFKFB3-driven glycolysis, which promotes EC proliferation and migration, CPT1a-controlled FAO selectively stimulates EC proliferation $[3,4]$.

Besides its importance in blood vascular ECs, CPT1a is also essential for lymphatic development [15]. Lymphatic ECs also rely on FAO to produce dNTPs to proliferate, similar to vascular ECs [15]. Notably, lymphatic EC metabolism, more specifically FAO, was shown to control the differentiation of lymphatic ECs through epigenetic mechanisms [15]. While it was known that the transcription factor PROX1 is essential to induce vascular to lymphatic EC differentiation, it was recently documented that PROX1 additionally stimulates FAO through binding to the CPT1a promoter, with a subsequent increase in CPT1a gene expression [15]. In fact, FAOderived acetyl-CoA is used by the histone acetyltransferase p300 for histone acetylation at PROX1-binding sites, preferentially at lymphatic genes (e.g., VEGFR3), thereby inducing the proliferation, migration, and differentiation of lymphatic ECs. As such, PROX1 exploits FAO for epigenetic changes at lymphatic genes, making them more accessible to enhance its own transcriptional activity [15]. It is tempting to speculate that not only lymphatic ECs, but also vascular ECs use metabolic intermediates for the epigenetic modulation of angiogenesis, although this requires further study.

\section{Glutamine Metabolism Is Linked to Asparagine}

ECs sustain proliferation and vascular expansion through the use of glutamine [16,17], the most abundant circulating nonessential amino acid (NEAA) [18]. In fact, of all amino acids, glutamine is the one consumed the most by ECs [16]. In proliferating ECs, glutamine serves as an anaplerotic source of carbons to replenish the TCA cycle via glutaminase 1 (GLS1) to support protein and nucleotide synthesis $[16,17]$. Accordingly, both protein and nucleotide synthesis were impaired upon glutamine depletion in ECs, mTOR activity was decreased, and endoplasmic reticulum (ER) stress marker genes were increased [16]. Furthermore, glutamine is used to produce glutathione (GSH) for redox homeostasis, and glutamine depletion made ECs more susceptible to ROS-induced damage $[16,19]$. However, reports differ on whether ECs utilize glutamine for ATP synthesis $[16,17]$. Also, ECs metabolize glutamine for citrate production by means of reductive carboxylation. In in vitro and in vivo models, interference with glutamine metabolism restrains angiogenesis by affecting EC proliferation $[16,17]$ and migration [16]. The link between $\alpha-K G$ and asparagine appears to be particularly crucial, because angiogenesis, impaired by limitation of glutamine (or its metabolism), can be rescued by supplementation with $\alpha-K G$ plus asparagine [16], or with $\alpha-K G$ alone when asparagine is

Figure 2. Fatty Acids and Amino Acids Support Nucleotide and Protein Synthesis. (A) Proliferating endothelial cells (ECs) metabolize fatty acids to sustain the TCA cycle for nucleotide synthesis. Upon fatty acid uptake by FATPs, CPT1a transports fatty acids into mitochondria, where they are oxidized to acetyl-CoA, which is then used to sustain the TCA cycle, in conjunction with an anaplerotic substrate, to generate precursors of nucleotide synthesis.(B) ECs use the serine synthesis pathway (SSP), fueled by the glycolytic intermediate 3PG, to produce nucleotides. Serine is synthesized by PHGDH, converted to glycine, and contributes to one-carbon metabolism. Glutamine, a source of nitrogen for serine synthesis, replenishes the TCA cycle at the level of $\alpha-K G$, which is used for fatty acid or nucleotide biosynthesis. Glutamine is also used for the production of glutathione for redox homeostasis. Impaired EC proliferation upon glutamine depletion is rescued by supplementation with $\alpha-K G$ plus asparagine, or with $\alpha-K G$ when asparagine is acquired via macropinocytosis. Given a role in suppressing ER stress and reactivation of mTOR signaling in other cell types, asparagine might also function as a metabolic regulator in ECs. Abbreviations: 1C, one-carbon; 3PG, 3-phosphoglycerate; $\alpha$-KG, $\alpha$-ketoglutarate; AA, amino acid; Ac-CoA, acetyl CoA; Asn, asparagine; ASNS, asparagine synthetase; Asp, aspartate; CPT1a, carnitine palmitoyltransferase 1a; dNTPs, deoxyribonucleotides; ER, endoplasmic reticulum; FA-CoA, fatty acid-CoA; FAO, fatty acid $\beta$-oxidation; FAs, fatty acids; FATP, fatty acid transporter; glc, glucose; GLS1, glutaminase 1; gly, glycine; mTOR, mammalian target of rapamycin; OAA, oxaloacetate; PHGDH, phosphoglycerate dehydrogenase; rNTPs, ribonucleotides; ser, serine; Slc1a5, solute carrier family 1 (neutral amino acid transporter), member 5; TCA, tricarboxylic acid cycle. 


\section{Trends in Cell Biology}

acquired from the extracellular milieu through macropinocytosis [17]. Notably, silencing of asparagine synthetase (ASNS), the enzyme producing asparagine from glutamine-derived nitrogen and aspartate, also impairs EC proliferation [16], highlighting the importance of asparagine, regardless of whether it is taken up from the culture medium or de novo synthesized. Interestingly, increasing evidence suggests a role of asparagine as a signaling metabolite that senses metabolic fuel reserves and availability, and accordingly coordinates cellular homeostatic responses [20] (Figure 2C). Studies with conditional knockout mice lacking the endothelial ASNS gene or RAC1 gene (an essential component of the macropinocytosis pathway) will help to prove the relevance of these in vitro findings.

\section{Serine Metabolism}

Serine can be not only taken up by ECs, but also de novo synthesized from the glycolytic intermediate 3-phosphoglycerate (3PG) in a three-step enzymatic reaction, controlled by the rate-limiting enzyme phosphoglycerate dehydrogenase (PHGDH) [21]. Of note, glutamine metabolism allies with glycolysis for serine synthesis, since the latter process uses nitrogen from glutamate [21]. Serine can be interconverted with glycine and is thereby connected to one-carbon metabolism for redox homeostasis and nucleotide synthesis [22] (Figure 2C). $\mathrm{PHGDH}$ is critical for ECs, because endothelial loss of PHGDH causes severe lethal vascular defects, due to impaired EC proliferation and survival, in part resulting from defective heme synthesis (S. Vandekeere and P. Carmeliet, unpublished).

\section{Alternative Metabolism of Glucose}

ECs use additional metabolic pathways; however, their exact role is understudied in the context of angiogenesis, especially in vivo. For instance, glucose is not exclusively destined for glycolysis once phosphorylated by hexokinase (HK) to glucose-6-phosphate (G6P). Instead, ECs can use part of the G6P pool for glycogen storage through the use of glycogenic enzymes [23-25]. Glycogen could be particularly useful as an endogenous source of glucose when ECs are sprouting into glucose-deprived milieus. The fact that inhibition of glycogen phosphorylase (PYG), a rate-limiting enzyme for glycogen breakdown, impairs EC migration and viability supports this hypothesis [24]. However, the role of glycogenolysis for angiogenesis is still largely speculative.

G6P can also enter the oxidative branch of the pentose phosphate pathway (oxPPP) to generate ribulose-5-phosphate (Ru5P) and NADPH [26]. Ru5P is a precursor for ribose-5phosphate (R5P), which is used for nucleotide biosynthesis [26]. In addition to other functions, NADPH is essential for restoring glutathione from its oxidized form, GSSG, to its reduced form, GSH, a key antioxidant [26]. The non-oxPPP branch connects to two intermediates of glycolysis (fructose-6-phosphate, F6P and glyceraldehyde-3-phosphate, G3P) and yields R5P, but not NADPH [26]. The inhibition of oxPPP or non-oxPPP, controlled by the ratelimiting enzymes G6P dehydrogenase (G6PD) and transketolase (TKT), respectively, leads to compromised EC viability and migration $[24,27]$.

Another side branch of glycolysis, the hexosamine biosynthesis pathway (HBP), originates from F6P and produces UDP-N-acetylglucosamine (UDP-GIcNAc) for protein $\mathrm{N}$ - and O-glycosylation, using acetyl-CoA, ATP, glucose, glutamine, and uridine in the process [28]. Therefore, the HBP was suggested to function as a nutrient sensor that modulates angiogenesis, since the glycosylation status co-determines the functionality of angiogenic pathways, such as VEGFR2 and NOTCH1 $[29,30]$. 


\title{
Trends in Cell Biology
}

\begin{abstract}
Metabolism of Tumor Endothelium
Blood vessels promote multiple inflammatory and malignant diseases. Emerging evidence suggests that the metabolism of ECs in these disorders is perturbed. For reasons of brevity, we focus here on cancer, and refer readers to Box 1 for other vascular disorders (including diabetes). Cancer cells stimulate angiogenesis to supply oxygen and nutrients. However, unlike in healthy tissue, blood vessels in tumors are disorganized, excessive in number, and dysfunctional [31]. Indeed, due to its abnormal development, the tumor vasculature lacks the organized hierarchy of a normal vascular bed and instead comprises a chaotic network of structurally and functionally defective vessels [32]. In addition, tumor vessels are leaky, tortuous, and have an uneven vessel lumen. Therefore, blood flow is arbitrary and sluggish, and the poor perfusion renders the tumor hypoxic, which, together with a leaky and sticky endothelial barrier, promotes metastatic escape of cancer cells [33,34] (Figure 3A). Initial peculiarities of tumor EC metabolism have begun to emerge.
\end{abstract}

\section{Tumor ECs Are Hyperglycolytic, yet Retain Functional Mitochondria}

ECs in the tumor vasculature rely, even more than healthy ECs, on glycolysis for ATP production and display a hyperglycolytic phenotype, evidenced by enhanced expression of glucose transporter GLUT1 and the glycolytic activator PFKFB3 [35,36]. In addition, compared with healthy ECs, tumor ECs hyperactivate the pentose phosphate and serine biosynthesis pathways, used for nucleotide and biomass synthesis [35] (Figure 3B). Several signals in the tumor microenvironment (TME), including hypoxia and proinflammatory cytokines [35] or hormonal signaling [37], upregulate PKFBF3 [3]. These observations might have implications for cancer treatment. Indeed, lowering glycolysis in tumor ECs by restraining PFKFB3 function arrests their proliferation [3,38], decreases EC leakiness, tightens the vascular barrier, and restores perfusion, all signs of tumor vessel normalization [35] (Box 2). Importantly, the aim of such a therapeutic strategy is to lower glycolysis to the level detected in normal ECs, not to decrease it too much or completely eliminate glycolytic flux, because this induces EC death and tumor vessel disintegration, thereby facilitating cancer cell escape and metastasis [39]; thus, dosing PFKFB3-blocker therapy is essential.

Box 1. Metabolic Changes in EC Metabolism in Other Diseases

In pulmonary arterial hypertension, pulmonary arterial ECs (PAECs) are hyperproliferative, similar to tumor ECs [76]. PAEC metabolism is characterized by increased glycolysis, fatty acid biosynthesis, and glutamine metabolism [76,77]. In other vascular diseases (such as atherosclerosis), nitric oxide (NO)-mediated vasoprotection is impaired due to its decreased production. NO is produced through conversion of arginine to citrulline by endothelial nitric oxide synthase (eNOS). Upregulation of arginase and asymmetric dimethylarginine (AMDA) in atherosclerosis can impair NO synthesis in ECs. Indeed, arginase metabolizes arginine to urea and ornithine, thereby depleting the arginine pool [78,79], while AMDA is a competitive inhibitor of eNOS [80]. In diabetes, the elevated circulating glucose levels increase ROS production in ECs through auto-oxidation of glucose, hyperglycemia-activated NADPH oxidases, and mitochondrial malfunctioning. ROS also cause eNOS uncoupling, thereby exacerbating oxidative stress due to the production of superoxide and $\mathrm{ONOO}^{-}$. The oxidative stress induces oxidative damage in DNA, activation of polyADP-ribose polymerase-1 (PARP1) $[81,82]$ and ultimately inhibition of the glycolytic enzyme GAPDH through ribosylation. Eventually, glycolytic intermediates accumulate upstream of GAPDH, triggering an increase in the polyol pathway flux, and in hexosamine biosynthetic and glycation pathways as an alternative to process the glucose overload. The polyol pathway consumes NADPH and produces sorbitol [83], thereby impairing ROS scavenging and increasing oxidative stress; sorbitol conversion to fructose yields 3-deoxyglucosone as its side product, which can also be formed from glyceraldehyde-3-phosphate and dihydroxyacetone phosphate (accumulated glycolytic intermediates) together with methylglyoxal and glyoxal. These three aldehydes are highly reactive and disrupt the integrity of the endothelium through DNA and protein glycation [84-86], which leads to the formation of toxic advanced glycation end products. The negative impact of ROS in EC metabolism was also documented in ischemic heart disease, embolic events, aneurysms, and aorta dissection. ROS production results from compromised mitochondrial respiration in ischemia and embolic disease [87], and from the inflammatory process in aneurysm and aortic dissection [88]. 


\section{Trends in Cell Biology}
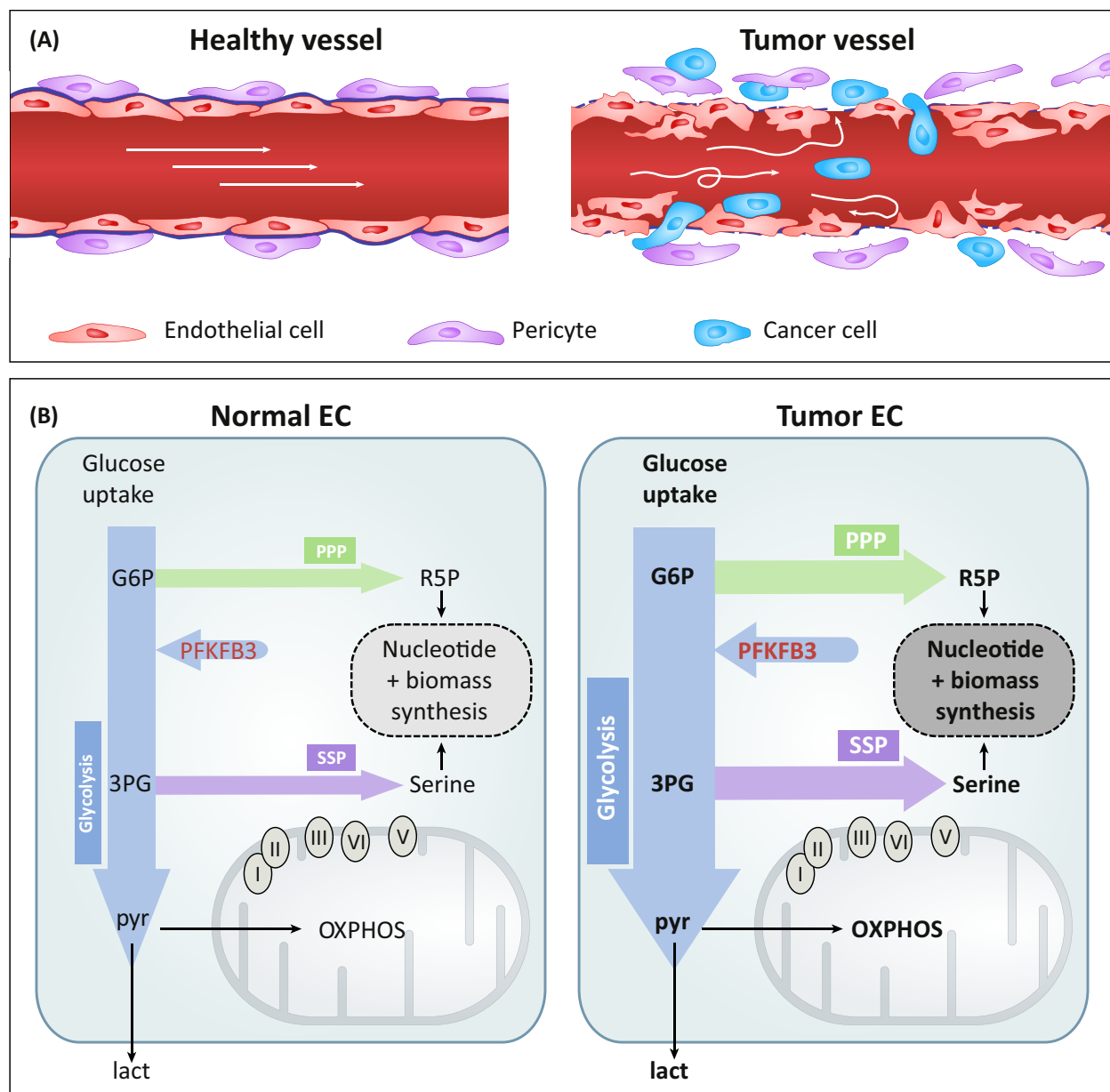

Trends in Cell Biology

Figure 3. Metabolism of Tumor Endothelial Cells (ECS). (A) Normal endothelium contains tightly adhering and interconnected ECs and an intact basement membrane supported by perivascular pericytes. Tumor endothelium lacks this organized hierarchy and comprises structurally and functionally defective vessels with leaky ECs, disrupted basement membrane, and poor pericyte coverage, leading to perturbed blood flow and facilitating the infiltration of cancer cells. (B) Normal proliferating ECs exhibit a high glycolytic flux triggered by activation of PFKFB3. The pentose phosphate pathway (PPP) and serine synthesis pathway (SSP) provide R5P and serine, respectively, as precursors for nucleotide and biomass synthesis. Tumor ECs are hyperglycolytic and present hyperactivity of the PPP and SSP, thereby sustaining the proliferative phenotype. Both healthy and tumor ECs retain functional OXPHOS. Abbreviations: 3PG, 3-phosphoglycerate; G6P, glucose-6-phosphate; lact, lactate; OXPHOS, oxidative phosphorylation; PFKFB3, phosphofructokinase-2/fructose-2,6-bisphosphatase 3; pyr, pyruvate; R5P, ribose-5-phosphate.

While glycolysis is crucial, tumor ECs retain functional mitochondria [40] (Figure 3B). Active OXPHOS not only adds to the flexibility of utilizing other substrates for energy production, but also provides metabolites for biomass synthesis to support proliferation, which may be of particular importance for the hyperproliferative tumor ECs. Accordingly, inhibition of mitochondrial respiration induced death of proliferating tumor ECs, more than of quiescent ECs [41-45]. The proposed mechanisms of cell death upon inhibition of mitochondrial respiration relate to the generation of ROS and uncoupling of the mitochondrial membrane potential. Therefore, the role of mitochondria in tumor ECs may be underestimated at present and warrants further investigation. 


\section{Trends in Cell Biology}

Box 2. Tumor Vessel Normalization

Traditional antiangiogenic therapy focuses primarily on the inhibition of VEGF signaling to prevent new vessel formation, thereby starving cancer cells. This strategy, although promising in preclinical trials [89], suffers from resistance and insufficient efficacy in patients with cancer [90]. An emerging alternative paradigm, tumor vessel normalization [91], aims to heal the structurally and functionally abnormal tumor vessels, thereby improving perfusion (and, hence, drug delivery), reducing hypoxia (which improves immune function), and tightening the vascular barrier (which impairs metastasis) [92]. Normalization of tumor vessels can be achieved by multiple approaches, including combined Tie2 receptor activation/ inhibition of angiopoietin 2 [93], chloroquine-induced activation of Notch1 signaling [94], and inhibition of angiotensin signaling [95]. This can also be achieved by inhibition of PFKFB3, which improves the efficacy of chemotherapy and reduces metastasis [96].

Glutamine and Fatty Acids in Tumor ECs

As discussed above, glutamine is essential for proliferation of ECs in vitro and in vivo [16]. Glutamine metabolism in ECs is induced by TGF- $\beta 1$ and RAF/MEK/ERK-signaling, pathways implicated in cancer progression [46]. Also, glutaminolysis is enhanced upon infection of ECs by Kaposi's sarcoma virus during the development of transformed lesions, and infected ECs become dependent on glutamine catabolism for their survival [47]. Such reports also suggest a role of glutamine metabolism in tumor ECs. Moreover, in contrast to cancer cells $[48,49]$, the proliferation defect of ECs upon glutamine starvation cannot be rescued by supplementation with antioxidants or replenishment of the TCA cycle alone, implying that ECs rely differently and perhaps also more prominently on glutamine, and additionally asparagine [16]. However, the exact role of glutamine/asparagine metabolism in tumor ECs is unknown.

Fatty acids are used by proliferating ECs to sustain the TCA cycle for de novo nucleotide synthesis during proliferation. These findings could also have therapeutic implications. Indeed, blockade of FAO inhibits injury-induced (lymph)-angiogenesis [15]. While these data suggest a therapeutic potential for FAO blockers to suppress tumor growth and metastasis, such an effect remains to be studied. Moreover, inhibitors of fatty acid synthase (FASN) decrease the viability, proliferation, and migration of lymphatic ECs, thereby impairing cancer cell spread [50].

\section{Metabolic Crosstalk between Endothelium and Other Cell Types}

While metabolism of individual cell types in the TME are a focus of intense research, how these various cell types communicate with each other via nutrient and metabolite exchange remains understudied. Cells in the TME experience selective pressure, in part due to the lack of nutrients and increased accumulation of metabolic waste products, caused to some extent by inefficient blood perfusion. These conditions alter the secretome of the cell, excreted directly or via exosomes [51].

ECs engage in metabolic crosstalk with both cancer and immune cells. An important factor in these interactions is local ischemia, determined by the position of a cell relative to the vasculature. This has been shown to affect the secretome of cancer cells, and to co-determine the differentiation of tumor-associated macrophages (TAMs), immune T cells, and ECs [52]. Also, cancer cells can prime ECs to proliferate by stimulating their glucose uptake [36], or by secreting metabolites, such as the TCA cycle intermediate succinate [53]. Another inducer of tumor EC proliferation is extracellular lactate, secreted in part by cancer cells [54] and cancerassociated fibroblasts [55]. Lactate acts as a signaling molecule and stimulates angiogenesis via enhancing VEGF signaling through the activation of HIF-1 $\alpha[56,57]$ and the PI3K/AKT pathway [58]. Moreover, ECs can take up lactate for oxidation upon conversion to pyruvate, which stimulates angiogenesis via NF-kB/L-8 signaling [59]. Lactate accumulation creates an acidic microenvironment, which causes ER stress in ECs and induces an inflammatory response [60]. 


\section{Trends in Cell Biology}

ECs in the TME interact with several immune cell types, which may evoke both pro- or antiangiogenic responses. For instance, type $1 \mathrm{~T}$ helper cells enhance tumor vessel normalization by changing the cytokine milieu and enhancing pericyte recruitment [61]. By contrast, macrophages have a dual role in the TME; although they can eliminate tumor cells, TAMs also act as drivers of tumor progression by promoting the proliferation of cancer cells and angiogenesis, eliciting an immunosuppressive environment [62]. In the TME, TAMs undergo changes in the expression of several metabolic genes to cope with hypoxia [63]. REDD1, a negative regulator of the key nutrient sensor mTOR [64], was shown to regulate the shared use of glucose between TAMs and ECs [65]. Indeed, inhibition of mTOR by REDD1 downregulates glycolysis in TAMs, which results in increased TME levels of glucose that can then be utilized by tumor ECs and promotes angiogenesis. Conversely, deletion of REDD1 induces glycolysis in TAMs, resulting in metabolic competition for glucose between TAMs and tumor ECs, which leads to decreased angiogenesis, normalization of tumor vessels, and reduced metastatic spread [65] (Figure 4).

The metabolism of immune cells relies on the subtype and activation status [66]. Hence, the lack of certain nutrients in the TME influences immune cell metabolism, which subsequently promotes the angiogenic phenotype of ECs but impairs immune cell activity $[67,68]$. For instance, TAMs in the TME express high levels of not only VEGF (which supports the proliferation of ECs) but also arginase 1, which reduces the levels of arginine in the TME [63], thereby impairing the function of $T$ cells [69]. The proangiogenic properties of TAMs are further dependent on glutamine synthetase. Inhibition of glutamine synthesis in TAMs decreases glutamine in the TME, leading to tumor vessel normalization and metastasis reduction [70]. By contrast, low glutamine levels in the TME, caused by cancer cells that switch to glutamine metabolism in response to acidification [71], negatively impact activated lymphocytes in the TME, which rely on glutamine as an energy substrate [72].

The network of metabolic interactions in a tumor becomes even more complex upon anticancer treatment, leading to compensatory mechanisms. For instance, glycolytic cancer cells in

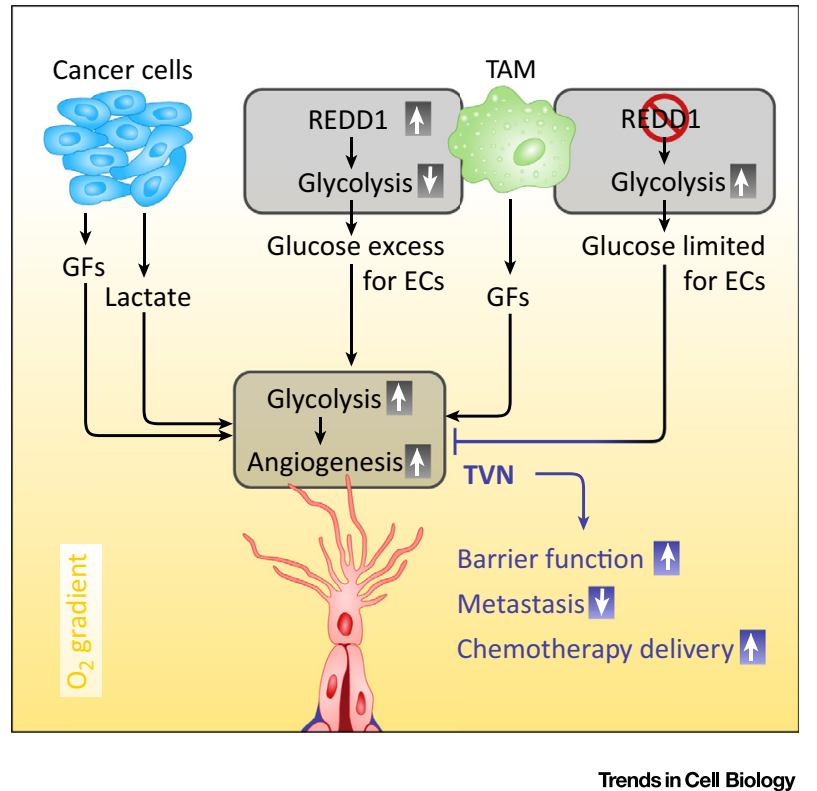

Figure 4. Metabolic Crosstalk of Endothelial Cells (ECs) in the Tumor Microenvironment. ECs and tumor blood vessels have a central role in establishing the tumor microenvironment and metabolic crosstalk of individual cell types. Accelerated angiogenesis of tumor ECs is stimulated by growth factors and lactate produced by cancer cells in poorly oxygenated areas. Tumor-associated macrophages (TAMs) in hypoxic regions upregulate the expression of REDD1, which hinders glycolysis in TAMs, thereby providing glucose for ECs. By contrast, blocking REDD1 increases glucose consumption by TAMs, leading to competition with ECs for glucose. Lower glucose availability for tumor ECs results in a lesshyperglycolytic phenotype and tumor vessel normalization. Abbreviations: GFs, growth factors; REDD1, regulated in development and DNA damage response 1; TVN, tumor vessel normalization. 


\section{Trends in Cell Biology}

CellPress REVIEWS hypoxic regions consume glucose and produce lactate, which is then used as substrate to fuel oxidative metabolism of neighboring cancer cells in the vicinity of blood vessels [73]. This metabolic symbiosis may cause resistance to antiangiogenic therapy, whereby cancer cells in more hypoxic regions feed cancer cells in more oxidative areas [74,75]. As another example, the antitumor effect of mTOR inhibitors is partially hindered by their effect on TAMs, as discussed above. Indeed, while inhibition of mTOR has antitumoral effects on cancer cells, it enhances the protumorigenic properties of TAMs by stimulating angiogenesis. Thus, simultaneous targeting of complementary pathways may improve the overall efficacy of therapeutic targeting of metabolism, but concerns about safety for such combination strategies will need to be addressed.

\section{Concluding Remarks}

EC metabolism is a key regulator of angiogenesis, and represents an attractive therapeutic target. Although overlooked for decades, substantial perturbations of the metabolism of tumor ECs and of the endothelium in other vascular diseases were recently documented. These differences between normal and diseased ECs might offer novel therapeutic opportunities for improved antiangiogenesis medication. Another level of opportunity relates to targeting metabolic crosstalk between ECs, cancer cells, and other stromal cells. The variety of metabolites in the environment, originating from several cell types, their integration for signaling, and functional outcome are yet to be fully determined. State-of-the-art technologies, such as single cell transcriptomics and metabolomics profiling, promise to yield exciting insights for future therapeutic approaches (see Outstanding Questions).

\section{Author Contributions}

All authors prepared and revised the manuscript, and approved the final version; K.R. and K.V. prepared the figures.

\section{Disclaimer Statement}

P.C. is named as an inventor on patent applications related to the results described in this manuscript.

\section{Acknowledgments}

The authors apologize to any author whose work could not be included due to space limitations. K.R. and K.V. are supported by the Research Foundation Flanders (FWO). The work of P.C. is supported by a Belgian Science Policy grant (IUAP7/03), long-term structural Methusalem funding by the Flemish Government, grants from the FWO (G.0834.13N and G.0532.10N), Foundation against Cancer (grant no. 2012-175), a European Research Council (ERC) Advanced Research Grant (EU ERC269073), and an AXA Research grant.

\section{References}

1. Potente, M. et al. (2011) Basic and therapeutic aspects of angiogenesis. Cell 146, 873-887

2. Jakobsson, L. et al. (2010) Endothelial cells dynamically compete for the tip cell position during angiogenic sprouting. Nat. Cell Biol. 12, 943-953

3. De Bock, K. et al. (2013) Role of PFKFB3-driven glycolysis in vessel sprouting. Cell 154, 651-663

4. Schoors, S. et al. (2015) Fatty acid carbon is essential for dNTP synthesis in endothelial cells. Nature 520, 192-197

5. De Bock, K. et al. (2013) Role of endothelial cell metabolism in vessel sprouting. Cell Metab. 18, 634-647

6. Helmlinger, G. et al. (2000) Formation of endothelial cell networks. Nature 405, 139-141

7. Yu, P. et al. (2017) FGF-dependent metabolic control of vascular development. Nature 545, 224-228

8. Doddaballapur, A. et al. (2015) Laminar shear stress inhibits endothelial cell metabolism via KLF2-mediated repression of PFKFB3. Arterioscler. Thromb. Vasc. Biol. 35, 137-145
9. Wilhelm, K. et al. (2016) FOXO1 couples metabolic activity and growth state in the vascular endothelium. Nature 529, 216-220

10. Betsholtz, C. (2016) Vascular biology: transcriptional control of endothelial energy. Nature 529, 160-161

11. Vander Heiden, M.G. et al. (2009) Understanding the Warburg effect: the metabolic requirements of cell proliferation. Science 324, 1029-1033

12. Neely, J.R. and Morgan, H.E. (1974) Relationship between carbohydrate and lipid metabolism and the energy balance of heart muscle. Annu. Rev. Physiol. 36, 413-459

13. Saddik, M. and Lopaschuk, G.D. (1991) Myocardial triglyceride turnover and contribution to energy substrate utilization in isolated working rat hearts. J. Biol. Chem. 266, 8162-8170

14. Kuo, A. et al. (2017) Lipid droplet biogenesis and function in the endothelium. Circ. Res. 120, 1289-1297

15. Wong, B.W. et al. (2017) The role of fatty acid beta-oxidation in lymphangiogenesis. Nature 542, 49-54

\section{Outstanding Questions}

Which other metabolic pathways are important for vessel sprouting?

In addition to vessel sprouting, other modes of vessel growth exist; are the same or different metabolic pathways involved?

ECs are a heterogeneous population of tip, stalk, and phalanx cells: does their metabolic signature differ, and how?

Arterial, venous, capillary, and lymphatic ECs in different organs differ from each other phenotypically, functionally, and morphologically; does their metabolism also differ?

Tumor ECs are hyperglycolytic, but which other metabolic pathways are perturbed?

Tumor ECs are heterogeneous; can their metabolic signature be profiled by single cell RNA sequencing and analysis of metabolic genes?

ECs become dysfunctional in diabetes; which other metabolic perturbations underlie EC dysfunction and can they be targeted?

Computational genome-scale metabolic modeling (GEM) allows one to predict metabolic candidates driving EC biology; will the development of EC-tailored GEMs accelerate the discovery of EC metabolic targets?

Will it be feasible to develop effective, yet safe antiangiogenic therapies based on targeting metabolic candidates, and will adequate dosing be possible?

Can other metabolism targeting-based strategies be developed to induce tumor vessel normalization?

Can the manipulation of metabolism or delivery of metabolites be used to stimulate (lymph)-angiogenesis for regenerative medicine or treatment of ischemic disease? 


\section{Trends in Cell Biology}

16. Huang, H. et al. (2017) Role of glutamine and interlinked asparagine metabolism in vessel formation. EMBO J. 36, 2334-2352

17. Kim, B. et al. (2017) Glutamine fuels proliferation but not migration of endothelial cells. EMBO J. 36, 2321-2333

18. Mayers, J.R. and Vander Heiden, M.G. (2015) Famine versus feast: understanding the metabolism of tumors in vivo. Trends Biochem. Sci. 40, 130-140

19. DeBerardinis, R.J. and Cheng, T. (2010) Q's next: the diverse functions of glutamine in metabolism, cell biology and cancer. Oncogene 29, 313-324

20. Zhang, J. et al. (2014) Asparagine plays a critical role in regulating cellular adaptation to glutamine depletion. Mol. Cell 56, 205-218

21. Amelio, I. et al. (2014) Serine and glycine metabolism in cancer. Trends Biochem. Sci. 39, 191-198

22. Tibbetts, A.S. and Appling, D.R. (2010) Compartmentalization of mammalian folate-mediated one-carbon metabolism. Annu. Rev. Nutr. 30, 57-81

23. Roach, P.J. et al. (2012) Glycogen and its metabolism: some new developments and old themes. Biochem. J. 441, 763-787

24. Vizan, P. et al. (2009) Characterization of the metabolic changes underlying growth factor angiogenic activation: identification of new potential therapeutic targets. Carcinogenesis 30, 946-952

25. Amemiya, T. (1983) Glycogen metabolism in the capillary endothelium. Electron histochemical study of glycogen synthetase and phosphorylase in the pecten capillary of the chick. Acta Histochem. 73, 93-96

26. Riganti, C. et al. (2012) The pentose phosphate pathway: an antioxidant defense and a crossroad in tumor cell fate. Free Radic. Biol. Med. 53, 421-436

27. Zhang, Z. et al. (2000) High glucose inhibits glucose-6-phosphate dehydrogenase via CAMP in aortic endothelial cells. J. Biol. Chem. 275, 40042-40047

28. Slawson, C. et al. (2010) O-GlcNAc signaling: a metabolic link between diabetes and cancer? Trends Biochem. Sci. 35, 547-555

29. Markowska, A.I. et al. (2011) Galectin-3 protein modulates cell surface expression and activation of vascular endothelial growth factor receptor 2 in human endothelial cells. J. Biol. Chem. 286, 29913-29921

30. Benedito, R. et al. (2009) The notch ligands DII4 and Jagged1 have opposing effects on angiogenesis. Cell 137, 1124-1135

31. Hanahan, D. and Weinberg, R.A. (2011) Hallmarks of cancer: the next generation. Cell 144, 646-674

32. Carmeliet, P. and Jain, R.K. (2011) Molecular mechanisms and clinical applications of angiogenesis. Nature 473, 298-307

33. DeClerck, K. and Elble, R.C. (2010) The role of hypoxia and acidosis in promoting metastasis and resistance to chemotherapy. Front. Biosci. (Landmark Ed) 15, 213-225

34. Jain, R.K. (2014) Antiangiogenesis strategies revisited: from starving tumors to alleviating hypoxia. Cancer Cell 26, 605-622

35. Cantelmo, A.R. et al. (2016) Inhibition of the glycolytic activator PFKFB3 in endothelium induces tumor vessel normalization, impairs metastasis, and improves chemotherapy. Cancer Cell 30, 968-985

36. Yeh, W.L. et al. (2008) Enhancement of glucose transporter expression of brain endothelial cells by vascular endothelial growth factor derived from glioma exposed to hypoxia. Mol. Pharmacol. 73, 170-177

37. Trenti, A. et al. (2017) The glycolytic enzyme PFKFB3 is involved in estrogen-mediated angiogenesis via GPER1. J. Pharmacol. Exp. Ther. 361, 398-407

38. Schoors, S. et al. (2014) Partial and transient reduction of glycolysis by PFKFB3 blockade reduces pathological angiogenesis. Cell Metab. 19, 37-48

39. Conradi, L.C. et al. (2017) Tumor vessel disintegration by maximum tolerable PFKFB3 blockade. Angiogenesis 20, 599-613

40. Koziel, A. et al. (2012) The influence of high glucose on the aerobic metabolism of endothelial EA.hy926 cells. Pflugers Arch. 464, 657-669
41. Blecha, J. et al. (2017) Antioxidant defense in quiescent cells determines selectivity of electron transport chain inhibitioninduced cell death. Free Radic. Biol Med. 112, 253-266

42. Coutelle, O. et al. (2014) Embelin inhibits endothelial mitochondrial respiration and impairs neoangiogenesis during tumor growth and wound healing. EMBO Mol. Med. 6, 624-639

43. Rohlena, J. et al. (2011) Mitochondrially targeted alpha-tocopheryl succinate is antiangiogenic: potential benefit against tumor angiogenesis but caution against wound healing. Antioxid. Redox Signal. 15, 2923-2935

44. Don, A.S. et al. (2003) A peptide trivalent arsenical inhibits tumor angiogenesis by perturbing mitochondrial function in angiogenic endothelial cells. Cancer Cell 3, 497-509

45. Orecchioni, S. et al. (2015) The biguanides metformin and phenformin inhibit angiogenesis, local and metastatic growth of breast cancer by targeting both neoplastic and microenvironment cells. Int. J. Cancer 136, E534-E544

46. Guo, Y. et al. (2016) Glutaminolysis was induced by TGF-beta1 through PP2Ac regulated Raf-MEK-ERK signaling in endothelia cells. PLoS One 11, e0162658

47. Sanchez, E.L. et al. (2015) Latent KSHV infected endothelial cells are glutamine addicted and require glutaminolysis for survival. PLoS Pathog. 11, e1005052

48. van den Heuvel, A.P. et al. (2012) Analysis of glutamine dependency in non-small cell lung cancer: GLS1 splice variant GAC is essential for cancer cell growth. Cancer. Biol. Ther. 13, $1185-1194$

49. Son, J. et al. (2013) Glutamine supports pancreatic cancer growth through a KRAS-regulated metabolic pathway. Nature 496, 101-105

50. Bastos, D.C. et al. (2017) Effects of fatty acid synthase inhibitors on lymphatic vessels: an in vitro and in vivo study in a melanoma model. Lab. Invest. 97, 194-206

51. Zhao, H. et al. (2016) Tumor microenvironment derived exosomes pleiotropically modulate cancer cell metabolism. Elife 5 , e10250

52. Carmona-Fontaine, C. et al. (2017) Metabolic origins of spatial organization in the tumor microenvironment. Proc. Natl. Acad. Sci. U. S. A. 114, 2934-2939

53. Garrigue, P. et al. (2017) The evolving role of succinate in tumo metabolism: an 18F-FDG-based study. J. Nucl. Med. Published online June 15, 2017. http://dx.doi.org/10.2967/ jnumed.117.192674

54. Polet, F. and Feron, O. (2013) Endothelial cell metabolism and tumour angiogenesis: glucose and glutamine as essential fuels and lactate as the driving force. J. Intern. Med. 273, 156-165

55. Zhang, D. et al. (2015) Metabolic reprogramming of cancerassociated fibroblasts by IDH3alpha downregulation. Cell Rep. $10,1335-1348$

56. Hunt, TK et al (2007) Aerobically derived lactate stimulates revascularization and tissue repair via redox mechanisms. Antioxid. Redox Signal. 9, 1115-1124

57. Porporato, P.E. et al. (2012) Lactate stimulates angiogenesis and accelerates the healing of superficial and ischemic wounds in mice. Angiogenesis 15, 581-592

58. Ruan, G.X. and Kazlauskas, A. (2013) Lactate engages recepto tyrosine kinases Axl, Tie2, and vascular endothelial growth factor receptor 2 to activate phosphoinositide 3-kinase/Akt and promote angiogenesis. J. Biol. Chem. 288, 21161-21172

59. Vegran, F. et al. (2011) Lactate influx through the endothelial cell monocarboxylate transporter MCT1 supports an NF-kappaB/I 8 pathway that drives tumor angiogenesis. Cancer Res. 71 2550-2560

60. Dong, L. et al. (2017) Acidosis activates endoplasmic reticulum stress pathways through GPR4 in human vascular endothelia cells. Int. J. Mol. Sci. 18, E278

61. Tian, L. et al. (2017) Mutual regulation of tumour vessel normallzation and immunostimulatory reprogramming. Nature 544, $250-254$ 


\section{Trends in Cell Biology}

62. Casazza, A. et al. (2013) Impeding macrophage entry into hypoxic tumor areas by Sema3A/Nrp1 signaling blockade inhibits angiogenesis and restores antitumor immunity. Cancer Cell 24 695-709

63. Colegio, O.R. et al. (2014) Functional polarization of tumourassociated macrophages by tumour-derived lactic acid. Nature 513, 559-563

64. Laplante, M. and Sabatini, D.M. (2012) mTOR signaling in growth control and disease. Cell 149, 274-293

65. Wenes, M. et al. (2016) Macrophage metabolism controls tumor blood vessel morphogenesis and metastasis. Cell Metab. 24, $701-715$

66. Buck, M.D. et al. (2017) Metabolic instruction of immunity. Cell $169,570-586$

67. Langston, P.K. et al. (2017) Metabolism supports macrophage activation. Front. Immunol. 8, 61

68. O'Neill, L.A. et al. (2016) A guide to immunometabolism for immunologists. Nat. Rev. Immunol. 16, 553-565

69. Rodriguez, P.C. et al. (2004) Arginase I production in the tumor microenvironment by mature myeloid cells inhibits $\mathrm{T}$-cell receptor expression and antigen-specific T-cell responses. Cancer Res. 64, 5839-5849

70. Palmieri, E.M. et al. (2017) Pharmacologic or genetic targeting of glutamine synthetase skews macrophages toward an M1-like phenotype and inhibits tumor metastasis. Cell Rep. 20, 1654-1666

71. Corbet, C. et al. (2014) The SIRT1/HIF2alpha axis drives reductive glutamine metabolism under chronic acidosis and alters tumor response to therapy. Cancer Res. 74, 5507-5519

72. Wang, R. et al. (2011) The transcription factor Myc controls metabolic reprogramming upon T lymphocyte activation. Immunity $35,871-882$

73. Sonveaux, P. et al. (2008) Targeting lactate-fueled respiration selectively kills hypoxic tumor cells in mice. J. Clin. Invest. 118, 3930-3942

74. Allen, E. et al. (2016) Metabolic symbiosis enables adaptive resistance to anti-angiogenic therapy that is dependent on $\mathrm{mTOR}$ signaling. Cell Rep. 15, 1144-1160

75. Pisarsky, L. et al. (2016) Targeting metabolic symbiosis to overcome resistance to anti-angiogenic therapy. Cell Rep. 15, $1161-1174$

76. Boucherat, O. et al. (2017) The cancer theory of pulmonary arterial hypertension. Pulm. Circ. 7, 285-299

77. Assad, T.R. and Hemnes, A.R. (2015) Metabolic dysfunction in pulmonary arterial hypertension. Curr. Hypertens. Rep. 17, 20

78. Nolan, D.J. et al. (2013) Molecular signatures of tissue-specific microvascular endothelial cell heterogeneity in organ maintenance and regeneration. Dev. Cell 26, 204-219

79. Stroes, E. et al. (1998) Origin of superoxide production by endothelial nitric oxide synthase. FEBS Lett. 438, 161-164
80. Napoli, C. et al. (2006) Nitric oxide and atherosclerosis: an update. Nitric Oxide 15, 265-279

81. Sawada, N. et al. (2014) Endothelial PGC-1alpha mediates vas cular dysfunction in diabetes. Cell Metab. 19, 246-258

82. Wang, X.R. et al. (2011) AMP-activated protein kinase rescues the angiogenic functions of endothelial progenitor cells via manganese superoxide dismutase induction in type 1 diabetes. Am. J. Physiol. Endocrinol. Metab. 300, E1135-E1145

83. Lorenzi, M. (2007) The polyol pathway as a mechanism for diabetic retinopathy: attractive, elusive, and resilient. Exp. Diabetes Res. 2007, 61038

84. Wautier, J.L. and Schmidt, A.M. (2004) Protein glycation: a firm link to endothelial cell dysfunction. Circ. Res. 95, 233-238

85. Bouraijaj, M. et al. (2003) Role of methylglyoxal adducts in the development of vascular complications in diabetes mellitus. Biochem. Soc. Trans. 31 (Pt 6), 1400-1402

86. Shinohara, M. et al. (1998) Overexpression of glyoxalase-l in bovine endothelial cells inhibits intracellular advanced glycation endproduct formation and prevents hyperglycemia-induced increases in macromolecular endocytosis. J. Clin. Invest. 101, $1142-1147$

87. Paternotte, E. et al. (2008) Review: behaviour of endothelial cells faced with hypoxia. Biomed. Mater. Eng. 18, 295-299

88. McCormick, M.L. et al. (2007) Role of oxidative stress in the pathogenesis of abdominal aortic aneurysms. Arterioscler. Thromb. Vasc. Biol. 27, 461-469

89. Kim, K.J. et al. (1993) Inhibition of vascular endothelial growth factor-induced angiogenesis suppresses tumour growth in vivo. Nature 362, 841-844

90. Jain, R.K. et al. (2006) Lessons from phase III clinical trials on antiVEGF therapy for cancer. Nat. Clin. Pract. Oncol. 3, 24-40

91. Jain, R.K. (2001) Normalizing tumor vasculature with anti-angiogenic therapy: a new paradigm for combination therapy. Nat. Med. 7, 987-989

92. Carmeliet, P. and Jain, R.K. (2011) Principles and mechanisms of vessel normalization for cancer and other angiogenic diseases. Nat. Rev. Drug Discov. 10, 417-427

93. Park, J.S. et al. (2016) Normalization of tumor vessels by Tie2 activation and Ang2 inhibition enhances drug delivery and produces a favorable tumor microenvironment. Cancer Cell 30 953-967

94. Maes, H. et al. (2014) Tumor vessel normalization by chloroquine independent of autophagy. Cancer Cell 26, 190-206

95. Chauhan, V.P. et al. (2013) Angiotensin inhibition enhances drug delivery and potentiates chemotherapy by decompressing tumour blood vessels. Nat. Commun. 4, 2516

96. Cruys, B. et al. (2016) Glycolytic regulation of cell rearrangement in angiogenesis. Nat. Commun. 7, 12240 